\title{
A Review of Intelligent Contactor Design and Its Control Technology
}

\author{
Ming Zong ${ }^{1}$, Lei Qi ${ }^{1,2}$, Xuejie Wang ${ }^{3,4}$, Ying Zhang ${ }^{4}$ and Shenghui Wang ${ }^{4}$ \\ ${ }^{1}$ Shenyang University of Technology, Shenyang 110178, China \\ ${ }^{2}$ Liaoning Provincial Institute of Safety Science, Shenyang 110004, China \\ ${ }^{3}$ Harbin Institute of Technology, Harbin 150001, China \\ ${ }^{4}$ Shenyang Institute of Engineering, Shenyang 110136, China \\ qileinedu@126.com
}

\begin{abstract}
The building of distributed power and smart grid accelerates the development of intelligent contactor. The design of intelligent contactor has gradually attracted more attention. This review introduces the latest research progress of intelligent contactor including four aspects such as intelligent design and optimization, no-arc control technology, less bounce control technology and subsidiary structure and function. This review focuses on power electronics technology, artificial intelligence content algorithm, and feedback control and so on. Some ideas are proposed to be further studied. Finally, the intelligent contactor's development is looked into with the future of China's energy management pattern.
\end{abstract}

Keywords: Intelligent; Optimization; Break; Bounce; Closed loop control, Switching power supply

\section{Introduction}

Currently, the electric power system is undergoing an unprecedented great change. On the one hand, from the point of the power source, the human concern on the depletion of fossil energy, energy security and environmental degradation. Energy conservation and emissions reduction, developing smart grid, and making full use of renewable energy have become the main development goals of power industry [1]. On the other hand, from the load side, with the advent of digital time, the electrical load contains a large number of electronic components, such as power electronic devices and computing control system, etc. So a new power system age is coming marked by new energy power, high precision and controllability.

The main problem of renewable energy power generation is the complexity of its control. For example, wind turbine should guarantee to generate qualified voltage waveform, as well as, it also has certain adaptability to the outside power interference. So now the primary concern of large-scale renewable energy power generation is to prevent disconnection caused by its own or external factors. Considering that the renewable energy makes up a growing percentage in the power system, the state advocates the new develop pattern of renewable energy, including distributed development, low-voltage access, and real consumption. At this point, some low voltage contactor's quality would affect the operation of the new energy power generation system directly. At present, most of wind turbines using AC690V contactors. The operation reliability of the contactor is put forward higher requirements for preventing wind generator stop [2].

In addition, the sensitivity to power quality is high of precision electronic components load equipment, so power quality would become a key assessment indicator. Improving the safe and reliable operation of the low-voltage distribution system is an important 
mission for low voltage contactor. Therefore, intelligent contactor's development becomes an inevitable trend.

Intelligent contactor is a new kind of control electric appliance. Digital processing chip or integrated circuit is adopted as the core of the intelligent control system in order to realize the contactor dynamic optimization control, including closing, holding and breaking process. Intelligent contactor is going to the direction of high reliability, energy saving, controllability, no noise and high voltage adaptation. Intelligent contactor with impact on power system will be enormous both in depth and width. At present, a lot of research work about intelligent contactor has been made.

This paper is to review the intelligent contactor research trend from multiple perspectives in recent years and focus on four aspects including intelligent contactor design and optimization, no arc breaking control technology, less closed bounce control technology, and accessory structure. Meanwhile, this paper provides references for subsequent further research work.

\section{Intelligent Contactor Design Optimization}

\subsection{Optimization Goal}

The goal of the traditional contactor structure design is single and independent. With the development of the intelligent contactor, design goal is diverse and the independence between goals is gradually weakened. Therefore, it is a trend to optimization design contactor by using modern multi-objective optimization design theory in the current study of the contactor product design [3]. The design goals of the intelligent contactor can be divided into two categories. One is the structural parameters, such as the choice of electromagnet height and excitation coil radius and so on, which consider on the perspective of cost saving. The other is the control parameters, such as contact closing time, closing phase angle and etc., which consider on realizing the ideal operation state. Text [4-8] all chose the core volume $\mathrm{V}_{\mathrm{Fe}}(\mathrm{x})$, coil volume $\mathrm{V}_{\mathrm{Cu}}(\mathrm{x})$, the impact energy of core-sectional per unit area $\mathrm{E}_{\mathrm{k}}(\mathrm{x})$, and electromagnetic closing time $\mathrm{T}(\mathrm{x})$. These parameters build a multi-objective function as follows.

$$
\min f(x)=\left[V_{F e}(x), V_{C u}(x), E_{k}(x), T(x)\right]^{T}
$$

There will be priorities in three goals. For example, if it is limited by design cost, $\mathrm{V}_{\mathrm{Fe}}(\mathrm{x})$ and $\mathrm{V}_{\mathrm{Cu}}(\mathrm{x})$ would be reducing. Therefore, weighted values and normalization processing is necessary to be build. The function after processing is extreme value function as follows.

$$
\min f(x)=\omega_{1}\left[\alpha \frac{V_{F e}(x)}{V_{F e}}+\beta \frac{V_{C u}(x)}{V_{C u 0}}\right]+\omega_{2} \frac{T(x)}{T_{0}}+\omega_{3} \frac{E_{k}(x)}{E_{k 0}}
$$

In the formula (2), $\mathrm{V}_{\mathrm{Fe} 0}$ and $\mathrm{V}_{\mathrm{Cu} 0}$ are objective function value before optimization, $\alpha$ and $\beta$ are the weight function of iron and copper amount, $\alpha+\beta=1, \omega_{1}, \omega_{2}, \omega_{3}$ are the weighted facto of each objective function.

\subsection{Optimization Method}

The selection of optimization method is to achieve the optimal value of extreme function. With artificial intelligence and multi-objective optimization method emerging constantly, some intelligent algorithms have been wildly used in the design and optimization of intelligent contactor.

a. Ant colony algorithm [4]

Ant colony algorithm was put forward by Italian scholars Dorigo.M for the first time. The idea comes from the ant's environment perception in the motive process, and the movement tends to the direction of high material intensity. 
Optimization variables such as maximum working air gap length, width of core and so on are defined, and it is equal to ants. The solve area can be divided into several small regions. The ants search mobile between the areas. The moving direction of a group of ants influenced by information is calculated. After a period of time, the small area of the objective function has the largest amount of information, and the optimal solution is obtained.

\section{b. Immune genetic algorithm [5]}

Immune genetic algorithm is a kind of improved genetic algorithm based on biological immune mechanism. The idea comes from the biological immune system generating the corresponding antigen to resist automatically.

Optimized variables are regarded as antibody. Antibody produced continuously is fitness calculated which is called genetic evolution. If the stopping amount of generation achieves stability, counting is stopped.

c. Neural network [6]

Neural network is a global optimization method. The training sample is established and optimized. The calculated optimal solution is considered as the center, and the size of the sample space is narrowed. The training samples increase around the narrowed area, and the simulation accuracy is improved. The characteristics of this method are to determine areas first, and then increase samples. The useless calculation on account of sample dispersion is reduced.

\section{d. Artificial fish algorithm [7]}

Artificial fish algorithm is similar to ant colony algorithm. Through dividing the grid, artificial fish moves to the grid. The value of the grid point is compared with bulletin board information, and the extreme grid point is determined. The scopes of the optimized variables are determined near the grid point. The grid is narrowed until the grid spacing achieves acceptable accuracy.

The above optimization algorithm has their characteristics respectively, but the main idea is similar. First the optimal areas are determined, and then other parts are ignored after determining the optimal area, while increasing the optimal sample in the area and then calculating [8]. However if the deviation of optimal area is big, it is difficult to return. Hence, how to rectify the fault area determined is a research direction in the future.

\section{No Arc Breaking Control Technology}

\subsection{Combined No Arc Contactor}

Generally, the type of contactor is point-contact. The arc in the breaking process leads to wear and fusion welding, which affects the life of the contactor directly. The contact material and control method need to continually be tested and improved, and seeking an alternative is a trend to improve the life and performance of contactor. The development of controlled power electronics device represented by thyristor provides the possibility. The less contactor and combined contactor are put forward recently. Combined contactor contains contact and non-contact two kinds of electrical appliances, which have the advantages of two kinds of electrical appliances at the same time. The advantages include high-voltage, over-current ability and no arc breaking. It is worth mentioning that because of the no arc breaking contactor with high safety performance, it can be replaced by explosion-proof electric apparatus in explosive dangerous field. Obvious economic benefits are obtained [9]. 


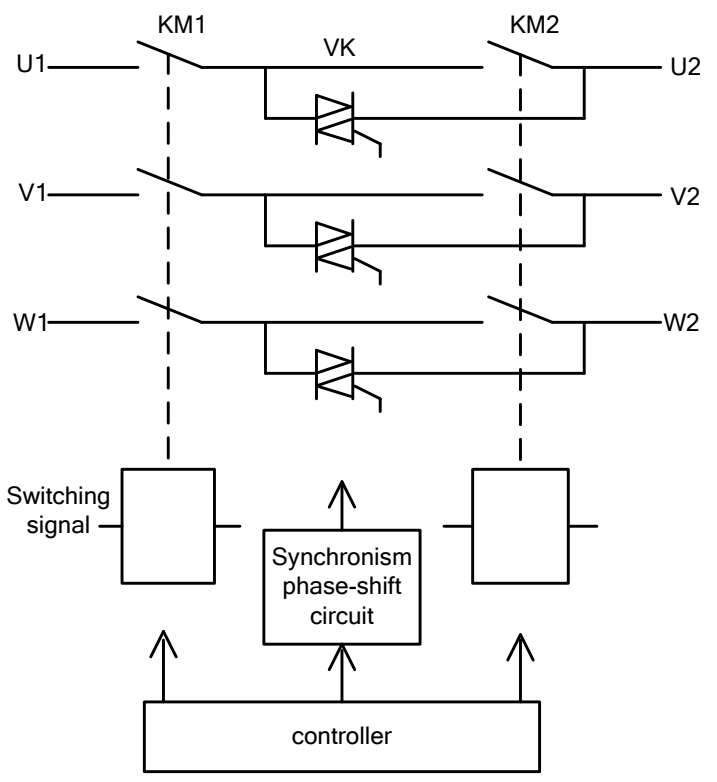

Figure 1. Combined Arc less Contacts

Figure 1 shows a main circuit diagram of modular arc-less contactor. KM is switch contact and $\mathrm{VK}$ is thyristor. As breaking the circuit, controller cuts off the power of KM2 coil. The KM2 voltage is 0 , and it is no-arc disconnect. VK thyristor's voltage is not zero after KM2 off. Making thyristor shut off by controlling trigger circuit. KM1 voltage is 0 and disconnected, then it is no-arc disconnect.

It can be seen that thyristor is the bridge of combined contactor no arc on and off. It makes two contacts contactor achieve arc less breaking freely when thyristor is on and off.

\subsection{Zero Current Breaking Control Principle}

Contactor breaking is zero current breaking essentially. Because the three phase's current is not zero at the same time, so it is difficult to control. Zhihong Xu and Peiming Zhang in the institute of electrical engineering and automation of Fuzhou university proposed breaking method at zero current in Three-phase perceptual load neutral ungrounded system [10-11].

As shown in Figure 2. Three phase current passes zero respectively. It is divided three areas on the timeline, which is the time difference of the three areas respectively. The three areas present the characters of periodic prosperity. For example, B phase current is the research object. When the B phase contact breaks in areaI, the B phase passes zero first, which is called the first breaking phase. If B phase disconnects successfully, A and $\mathrm{C}$ phase current are turned into line current. The breaking time of $\mathrm{AC}$ phase line current differs by $1 / 4$ cycle from B phase. So it is successful to disconnect AC line current after $5 \mathrm{~ms}$ when the B phase disconnected successfully. 


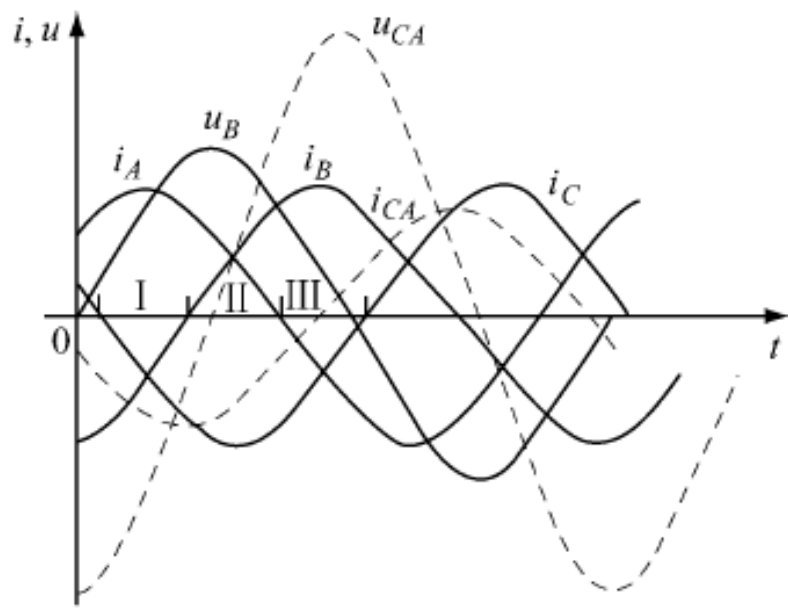

Figure 2. Symmetrical Voltage and Current Waveforms

\subsection{Zero Current Breaking Adaptive Control}

Text [12] proposed the best area of zero current breaking, which is usually $0.3 \mathrm{~ms}$ $0.4 \mathrm{~ms}$ earlier than the first phase passing zero time. This time mainly depends on the situation of the action of the electromagnetic mechanism, so it is uncertainty. Electromagnetic mechanism delay would make breaking point fall outside the best area, and cause the breaking failure.

Adaptive control is the adaptive changes of delay time for breaking command. The time is the delay time of the contactor receiving the orders that comes from software. If breaking failure, the current arc does not go out. At this time the delay time is modified, until the breaking point falls on the best breaking area.

In addition, text [13] proposed to apply magnetic retainer instead of contactor itself to give full application of short action time and self-hold function etc., which has a certain application prospect.

\subsection{The Breaker Dynamic Model Based On Neural Network}

The intelligent ac contactor breaking dynamic model based on neural network considers contact breaking speed and induction voltage in the breaking process. The faster contact breaks, the stronger ability of fast breaking circuit. The severe change of magnetic flux leads to higher induction voltage at the same time, which influences the coil insulation. Therefore, to seek the best breaking speed is the key to the optimization design.

Because the contactor breaking process is relatively complicated, especially the breaking process of magnetic state change is difficult to use mathematical model to represent. So optimization method based on mathematical model can not be used.

Text [14] proposed variation curve fitting method of excitation coil induction voltage based on artificial neural network in contactor breaking process. The method is based on multiple experiments, and then builds induction voltage curve library. Through the methods of artificial and fitting, prediction model of induction voltage is put forward with the experimental sample in breaking process. The method improves safety performance and dynamic character in the breaking process of intelligent contactor.

Because of the sampling device, the bad data existing in the real-time numerical got by the method based on the experiment is inevitably. Therefore, how to eliminate the bad data and correct the occasional mistake is a problem that should be considered, which can be a research direction in the future. The theory of power system state estimation is one of the solutions to the problem [15]. 


\section{Contact Bounce Elimination Technology}

\subsection{The Dynamic Process of Contactor Closing}

The closing reliability of Electromagnetic mechanism depends on whether the suction characteristic matches the anti-force characteristic or not [16]. Because of collisions between contacts and iron core by the force, the contact bounce is inevitable during the process of the contactor closing. On the one hand, the bounce intensifies the wear of the contact and shortens the mechanical life. On the other hand, the arc in the process of bouncing will also bring influence to the electrical life. Therefore, how to reduce the contact bounce of the contactor in the closing process has become a research hotpot recently. A lot of scholars have proposed many effective control methods.

Researching contactor closing process is the basis for the control of contact bounce. According to the relevant literatures of recent years, the method of studying the process of closing contains mathematical model analysis and simulation analysis.

a. Mathematical model analysis [17]

DC electromagnetic contactor, for example, its mathematical model shown as follows.

$$
\left\{\begin{array}{l}
\frac{d \Psi}{d t}=u-i r+u_{0} \\
\frac{d v}{d t}=\frac{F_{x}-F_{f}}{m} \\
\frac{d x}{d t}=v
\end{array}\right.
$$

In the formula (3), $\Psi$ is magnetic flux, $\mathrm{U}$ is coil excitation voltage, $\mathrm{i}$ is coil current, $\mathrm{r}$ is coil resistance, $\mathrm{u}_{\mathrm{o}}$ is holding voltage, $\mathrm{V}$ is movement speed of the core, Fx is electromagnetic suction, Ff is anti-force, $\mathrm{m}$ is electromagnetic mass, and $\mathrm{x}$ is the shift of the moving parts.

The formula (3) uses fourth order Runge-Kutta method to solve generally. The essence of equations is to obtain ideal core movement speed by controlling the coil voltage, and to achieve the goal of less contact bounce.

Formula (3) is a set of multivariate equations apparently, and the solution method is complicated. In the process of movement, there are couplings between variables. For example, electrical parameters are affected by the movement displacement. Therefore, some scholars are prone to simulation research [18-19].

b. Simulation analysis

Text [20] proposed permanent magnet contactor dynamics simulation analysis based on ANSYS and ADAMS software. These two simulation software packages are typical simulation tools of electromagnetic mechanism.

According to the method of finite element, physical field analysis software ANSYS subdivides the model based on the Maxwell electromagnetic field theory. The boundary conditions of parallel and vertical are applied in the subdivision process.

Mechanical system dynamics simulation software ADAMS shows contactor structure components of each system, including core, contact, spring, etc. The main characteristic of the simulation tool is intuitive, which can reflect connection relationship of each part clearly. So it has been applied widely in product design and development.

The two methods introduced can be applied to design and research of the process of dynamic closing, and have theirs own advantages. Mathematical model establishes the model of electromagnetic mechanism fundamentally, and it is suit to explore the relationship between electric, magnetic, force, and movement of intelligent contactor. However, the simulation analysis is relatively straightforward and easy to understand. The advantages of methods can be combined in the future study. 


\subsection{Contactor Closing Time Control}

Controlling closing and bounce time is an important way to reduce the contact bounce [21].There is no specific theoretical reference on the bounce time control. However, the control method of closing time has experimental results published.

The way to control closing time of contactor is that compute the integral for the excitation coil sampling current, and it is compared with closing current effective value to determine whether closing time coming. When comes to the close time, and then holds. The excitation voltage and current, collisions are all reduced [22]. The current effective value can be expressed as follows.

$$
I=\sqrt{\frac{1}{T} \int_{0}^{T} i^{2} d t}
$$

The discrete style of formula (4) can be written as formula (5).

$$
I^{2}=\frac{1}{n} \sum_{k=0}^{n} i_{k}^{2}
$$

When the integral current is equal to the reference current I, the closing time comes. In order to reduce the error caused by the discrete sampling, processing chip with high sampling frequency can be used. Integrator and comparator build by analog circuit can also be used in order to realize real-time control, which is a research direction in the future.

\subsection{Dynamic Control of Intelligent Contactor Based on Feedback}

Feedback is an important way to achieve stability control system. Coil voltage or current is hoped to maintain stable to meet the dynamic stability control of intelligent contactor. Coil voltage or current is hoped to be greater than the closing value slightly in order to realize a soft landing of the contact.

The voltage [23-25] and current [26] feedback control of intelligent contactor is proposed by Yingyi-Liu and Degui-Chen in Xi'an Jiaotong University. For instance, block diagram of voltage feedback control is shown in Figure 3.

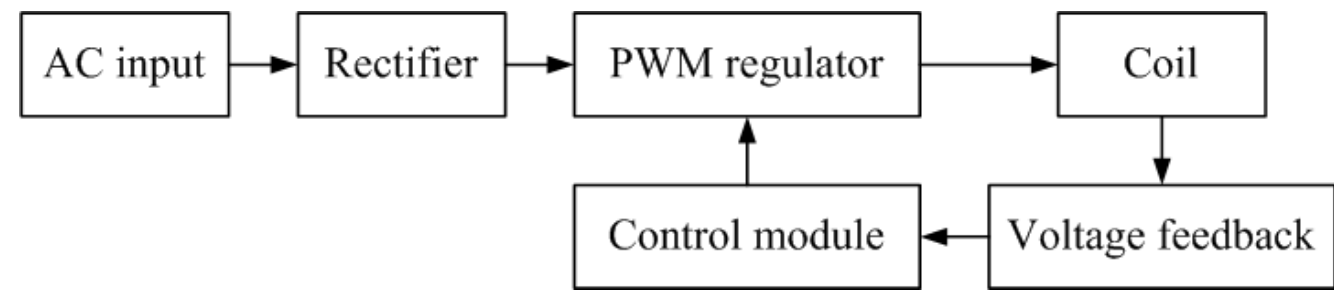

Figure 3. Voltage Feedback Control Block

After rectifier, the AC voltage into pulse width modulation (PWM) regulator is applied to contactor coil, and then feedbacks voltage to the control module. The module achieves the duty cycle control to keep the coil voltage unchanged. Insulated gate bipolar transistor (IGBT) is selected as the drive switch. The experimental results show that contact bounce time is shorter than ordinary AC contactor significantly. The principle of current feedback is the same as voltage feedback. Their difference is that error amplifier and PWM comparator is used in current feedback control module.

\subsection{The Determination of Variable Parameters in Closing Process of Contactor}

Contact closure is a dynamic process. As analyzed before, some parameters are timevarying. The electric circuit equation of contactor is shown as formula (6). 


$$
u(t)=R i+\frac{d(L i)}{d t}=R i+i \frac{d L}{d t}+L \frac{d i}{d t}
$$

It can be known that the coil inductance is variable, and the resistance is also variable according to the change of the environment. Therefore, the two variables should be definite in order to meet the accuracy of the control method.

A choice relatively close to the resistance value is calculated in practice by artificial learning method and building learning samples. Text [27] proposed a temperature compensation control strategy. The parameters at different temperatures are considered. The dynamic output control table is established through experiment. The table contains optimal PWM duty ratio and the excitation time at different voltage and temperature.

The Spanish Catalonia University Espinosa established the expression of resistance and inductance [28-29]. The expression shows that resistance and inductance can be calculated by real-time voltage, current, and their variation. The promotion and application of the expression would greatly reduce the cost of device, and the accuracy of the control method can be enhanced.

\subsection{Core Magnetic Flux Control Based On PWM}

The development of power electronic converter technology provides a broad platform for the research of intelligent contactor control method. By changing the power electronic switching frequency, the coil voltage and current change, and core flux can be adjusted [30-31]. Text [32-33] applied active inverter circuit to the flux control of the electromagnetic contactor. Core magnetic flux is strengthened and weakened by controlling coil voltage based on PWM technology. Core electromagnetic suction can be controlled. The main circuit topology is shown as Figure 4.

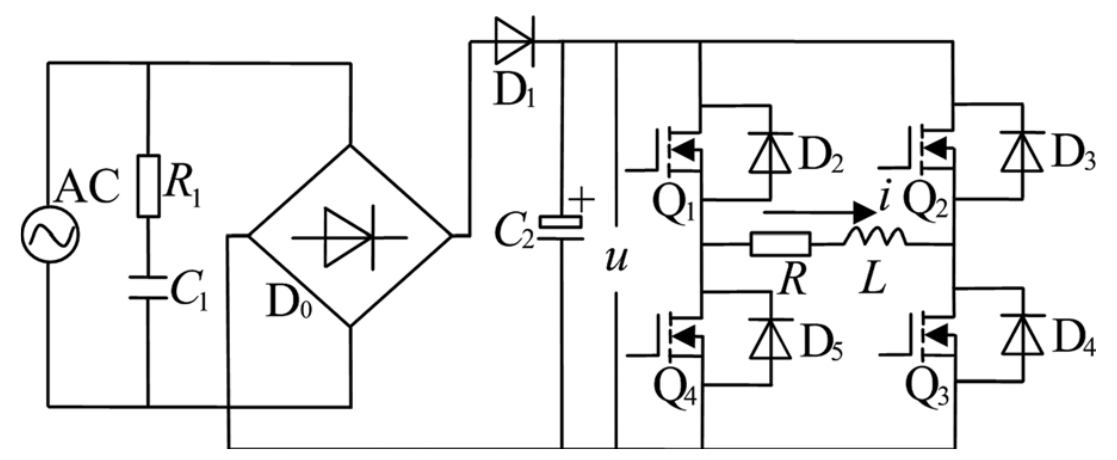

Figure 4. PWM Control of the Main Circuit

The AC power is converted to DC power by the uncontrolled rectifier. In the DC side, capacitor C2 and diode D1 are used for the stability of DC voltage and DC current. The single-phase bridge is in the inverter side, which is composed of four switches and parallel diodes. The switches are used to obtain the required PWM pulse voltage, and the parallel diodes are used for energy feedback. The control strategy diagram is shown as Figure 5 . 


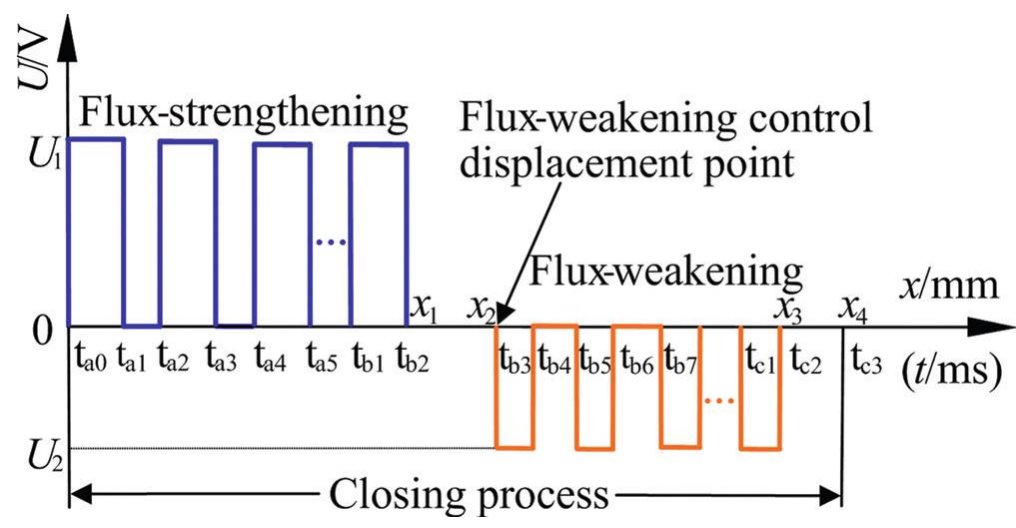

Figure 5. PWM Control Strategy

Keeping forward voltage stable in the process of strengthening magnetic flux makes the electromagnetic suction force bigger than anti-force. Keeping reverse voltage stable in the process of weaken magnetic flux makes electromagnetic suction reduce fast. The velocity of core closing and the impact force are also reduced, and the soft landing is achieved.

The circuit structure realizes the control of electromagnetic suction, and the control precision depends on the stability of the DC side voltage. The control strategies of PWM rectifier such as feed-forward decoupling control [34] and feedback linearization control [35] are applied in order to stable DC voltage rapidly.

\section{Accessory Structure and Function}

\subsection{Switch Power Technology}

Intelligent contactor must be accompanied by the improvement of the electromagnetic mechanism's power source. Switching power source usually serves power electronic technology, which also makes positive contribution to the development of intelligent contactor.

Switching power source is divided into four basic types, including voltage-type, current-type, charge-type and magnetic flux-type [36], but the fundamental is voltage control. Intelligent electric power usually involves rectifier (AC/DC), the inverter (DC/AC) and DC chopper (DC/DC) and other forms [36], and all kinds of circuits and the control methods have been reported. With the development of integrated circuits, various types of switching power source of integrated chip are also emerged.

Text [37] introduced three switching power source integrated chips based on TOPSwitch- II, LinkSwitch-TN and DPA-Switch. The characteristics of chips are wide input voltage, low power consumption, low electromagnetic interference, and protect function. The digital signal processor (DSP) has been widely used in the field of electric control [38]. The characteristic of DSP contains: $a$. high calculation speed, the chip can perform hundreds of millions of times per second to realize complex algorithm. $b$. The chip has abundant peripheral modules, including event manager (EV), A/D module, serial communication module (SCI) and so on, which have been applied to the development and design of switching power source at present. It will be a good choice of processing chip in the future.

\subsection{Accessory Control Module}

Intelligent contactor is a closed-loop control system actually. The safe and reliable operation also needs the support of all kinds of control module. 


\section{a. Detection module}

The coil voltage is sampled through sensors and the current is sampled by hall device. In order to meet the needs of the control chip processing, the sampling signal should be modulated. Text [39] introduced a kind of typical signal regulating circuit. The circuit consists of low signal and signal excursion.

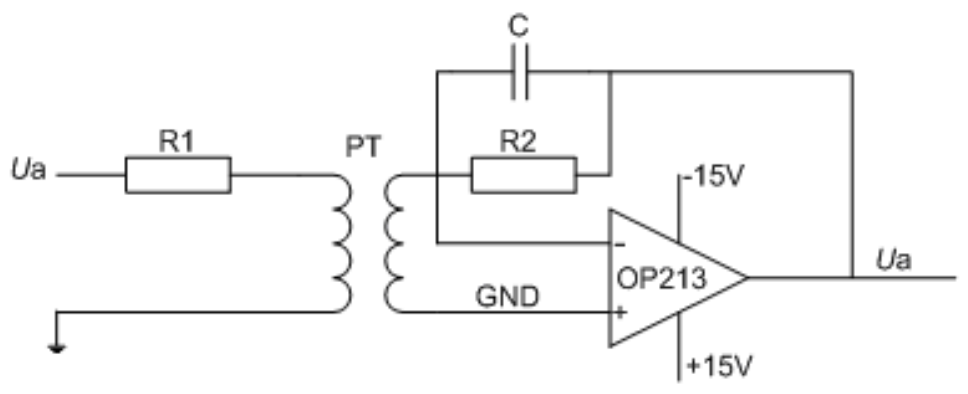

Figure 6. Signal Narrow Circuit

Figure 6 is a schematic of signal narrow. A small current is created by voltage acting at the resistant R1 in the primary winding side. After an induction to the secondary winding side of voltage transformer, a small voltage of resistance R2 is exported. Because the input voltage of control chip is positive, a migration signal is needed. The migration signal makes the whole signal rise up a value, keeping the voltage value of signal match the input voltage range of processing chip. The circuit structure is shown as Figure 7.

\section{b. The snubber circuit}

PWM control technology is used to control switch power source. When the contact breaks and the switch turn off, the energy of the coil is needed to be released. Therefore, the snubber circuit is designed. The snubber circuit has a lot of mature products. For example, Text [40] proposed the snubber circuit composed of electronic device and Darlington tube.

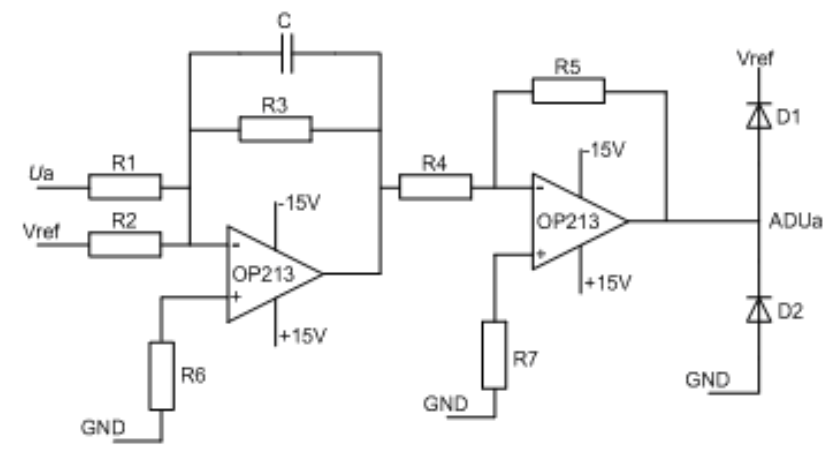

Figure 7. Signal Migration Circuit

\section{c. Resistance to voltage sags module}

The power system suffers to the external fault in operation processing, including lightning and short circuit causing voltage sags. Several voltage cycles sags can cause sensitive electrical equipment downtime, which damage the economic interests seriously [41].

The working characteristic of contactor is analyzed under the interference electricity fault in text [42]. Text [43] proposed voltage sags conversion function. The conversion principle diagram is shown in Figure 8. The alternating current flows through the contactor coil and normally closes contact when power on. When circuit failure or voltage sags is detected, driving switch stops. Alternating current is added to contactor coil directly. The contactor is still kept in close status. 


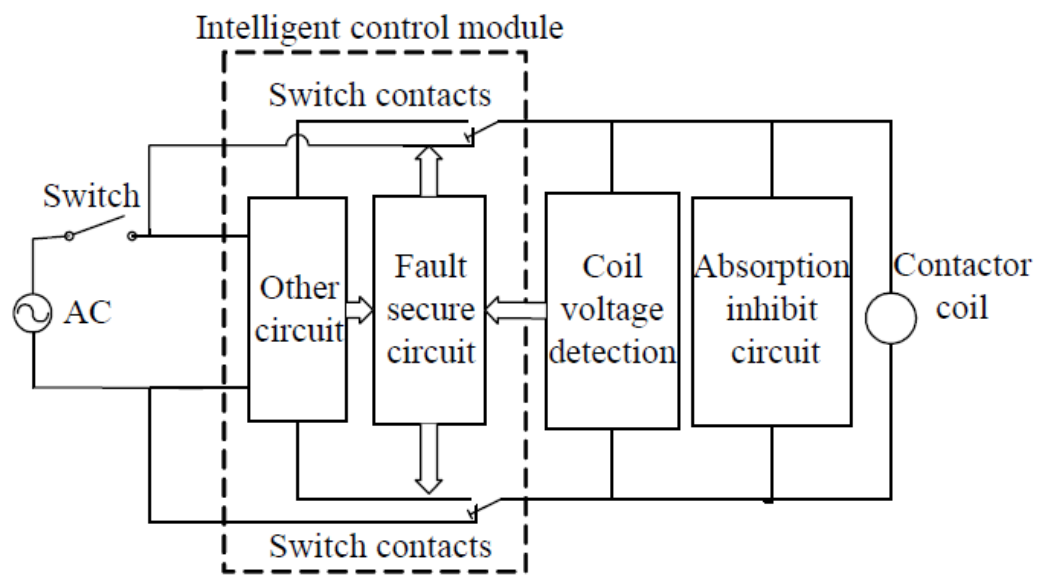

Figure 8. Failure Converting Schematic

The module actually proposed intelligent contactor voltage adaptability, which can be extended other power quality adaptability. When power quality event such as voltage sags, harmonic and unbalanced occurred, intelligent contactor can ensure the normal operation in a period of time. It will be an important future research direction. It will also contribute to the discussions of the intelligent contactor operational standards.

\section{Conclusion}

In this paper, the research results of intelligent contactor design and operation control are summarized in recent years. Four aspects are discussed systematically including intelligent contactor design optimization, no arc breaking control technology, contact bounce elimination technology and accessory structure and function.

Designing intelligent contactor is multi-objective. Cost is saved by modern optimization algorithm, and the operation mode is also optimized. Breaking without arc is solved through two kinds of methods, including non-contact and zero current. The adaptive control of the data and removing the bad data is beneficial to reliability of breaking without arc. Contact bounce can be eliminated by controlling time and feedback. The power electronic device provides a good platform for closing less bounce control. Intelligent contactor needs the support of each control module as a close loop control system. Power quality adaptability is an important symbol in the development of intelligent contactor.

Since the concept of the intelligent contactor is put forward, a lot of research progress has been got. Intelligent contactor conforms to the developing direction of power system in the future, which has a broad prospect. With the reform of the Chinese energy management and market, the development of the distributed power and smart grid, intelligent contactor will be able to take on the man-machine communication, adaptive, protection and optimal control, etc.

\section{Acknowledgements}

The authors gratefully acknowledged the support from National Natural Science Foundation of China (serial number: 51177105). 


\section{References}

[1] M. Ding, W. S. Wang and X. L. Wang, "A Review the Effect of Large-scale PV Generation on Power Systems", Proceedings of the CSEE, vol. 34, vo. 1, (2014).

[2] D. G. Chen, "Some Trends of Low Voltage Intelligent Electrical Apparatus", Electric industry, vol. 2, no. 1, (2009).

[3] Y. D. Cao, X. M. Liu and D. Liu, "Investigation of a Dynamic Neural Network Approach and Its Application of Multivariable Optimization", Proceedings of CSEE, vol. 26, no. 112 (2006).

[4] Z. H. Xu and P. M. Zhang, "Optimal Design of Intelligent AC Contactors Based on ant Colony Algorithm", Advanced Technology of Electrical Engineering and Energy, vol. 24, no. 9, (2005).

[5] L. Chen, P. M. Zhang and X. R. Miu, "Optimal Design of Intelligent Electromagnetic Devices based on Immune Genetic Algorithm", Advanced Technology of Electrical Engineering and Energy, vol. 22, no. $17,(\mathbf{2 0 0 3})$.

[6] Z. H. Xu and P. M. Zhang, "Dynamic Optimal Design of Intelligent AC Contactor in the Whole Course [J]. Proceedings of CSEE, 25, 156 (2005)

[7] D. W. Chen, P. M. Zhang. Virtual Prototype Optimal Design of Intelligent AC Contactors Based on Artificial Fish-Swarm Algorithm", Transactions of China Electro technical Society, vol. 26, no. 101, (2011).

[8] X. B. Wang, H. Y. Lin and S. H. Fang, "Design Optimization, and Intelligent Control of PermanentMagnet Contactor", IEEE Transaction on industrial electronics, vol. 60, no. 5148, (2013).

[9] A. A. Zhang, "The Research on the Switching without Arc for Contactor", Journal of Xiangtan Ming Institute, vol. 12, no. 35, (1997).

[10] Z. H. Xu and P. M. Zhang, "Zero Current Interrupt Control of Intelligent AC Contactor", Advanced Technology of Electrical Engineering and Energy, vol. 21, no. 54, (2002).

[11] Z. H. Xu and P. M. Zhang, "The Dynamic Breaking-Course Studying Adapt to Zero-Current-Break Control", Journal of Fuzhou University (Nature Science), vol. 34, no. 212, (2006).

[12] X. Zheng, Z. H. Xu and P. M. Zhang, "Analysis and Implementation of Zero-Breaking Adaptive Control in Intelligent AC Contactor", Advanced Technology of Electrical Engineering and Energy, vol. 24, no. 77, (2005).

[13] W. R. Li and X. J. Liu, "Micro-Arc Energy Breaking Technology of Intelligent AC Contactor Based on Magnetic Latching Relays", Electric appliance and energy management technology, vol. 2, no. 30, (2014).

[14] Z. H. Xu, P. M. Zhang and X. Zheng, "Realization of Zero-Current-Break Control Technology for Intelligent AC Contacator", Low-voltage apparatus, vol. 7, no. 6, (2006).

[15] X. J. Wang, L. Qi and D. F. Huang, "State Estimation and PMU Configuration Based on Wide Area Measuring System", Jilin Electric Power, vol. 38, no. 17, (2010).

[16] B. L. Wang, "Electromagnetic Electrical Design Basis", Beijing: The defense industry press, (1989).

[17] Z. H. Xu and P. M. Zhang, "Research on Dynamic Closing Course of Intelligent AC Contactor", Proceedings of CSEE, vol. 27, no. 108, (2007).

[18] Q. Wang, D. G. Chen and X. W. Li, "Synchronous Control of AC Contactor", Low-voltage apparatus, vol. 8 , no. 3, (2004).

[19] M. Z .Rong, J. Y. Lou and X. H.Wang, "Simulation Analysis and Experimental Research of Operating Characteristic for Moving Contact of Contactor with Permanent Magnet Actuator", Proceedings of CSEE, vol. 25, no. 109, (2005).

[20] J. Y.Lou, M. Z. Rong and H. C. Zou, "Simulation on Mechanism Dynamic Chrematistics of Single Coil and Single Stable Position Contactor with Permanent Magnet”, Proceedings of CSEE, vol. 24, no. 120, (2004).

[21] J. P. Chen, J. T. Hu and Q. Li, "Study of AC Contactor Bounce Control in Dynamic Process", Lowvoltage apparatus, vol. 19, no. 4, (2010).

[22] H. B. Xu, X. G. Zhu and G. C. Wu, "Research of Closing Time for Hybrid AC Contactors", Low-voltage apparatus, vol. 19, no. 10, (2013).

[23] Y. Y. Liu, D. G. Chen and C. P. Niu, "Analysis and Simulation of Dynamic Behavior and Contact Bounce for an Intelligent", Proceedings of the CSEE, vol. 27, no. 20, (2007).

[24] T. Shen, D. G. Chen and T. Feng, "Intelligent AC Contactor with Feedback Control", Low-voltage apparatus, vol. 2, no. 3, (2005).

[25] L. Ji, D. G.Chen and Y. Y. Liu, "Thermal Analysis of Contactors with Voltage Feedback System', Journal of Xi'an Jiaotong University, vol. 44, no. 90, (2010).

[26] Y. Y. Liu and D. G. Chen, "Simulation and Analysis on Dynamic Characteristics", Proceedomgs of the CSEE, vol. 30, no. 118, (2010).

[27] L. Zhou, L. Shu and G. C. Wu, "Conrtrol Strategy of Temperature Compensation of Intelligent AC contactors", Application of Electronic Technique, vol. 39, no. 73, (2013).

[28] A. G. Espinosa, J. R. Rida and J. Cusido, "Contact Bounce Elimination by Means of a Sensorless Closed-Loop Current Controller in DC Core Contactors", IEEE Transaction on industrial electronics, vol. 45 , no. $117,(\mathbf{2 0 0 9})$. 
[29] A. G. Espinosa, J. R, Rida and J. Cusido, "Closed-Loop Controller for Eliminating the Contact Bounce in DC Core contactors", IEEE Transaction on components and packaging technologies, vol. 33, no. 535, (2010).

[30] L. G. Tang and Z. H. Xu, "Design of an AC Contactor Module Started by Closed Loop Chopping", Proceedings of the CSEE, vol. 33, no. 132, (2013).

[31] X. B. Wang, H. Y. Lin and S. L. Ho, "Analysis of Dynamic Characterstics of permanent Magnet Contactor with Sensorless Displacement Profile Control", IEEE Transaction on magnetics, vol. 46, no. 1633, (2010)

[32] X. B. Wang, H. Y. Lin and S. H. Fang, "Analysis of Flux-weakening Control and Dynamic Characteristic for Sensorless Intelligent Permanent”, Proceedings of CSEE, vol. 31, no. 93, (2011).

[33] X. B. Wang, H. Y. Lin and S. H. Fang, "Dynamic Performance Analysis of Permanent Magnet Contactor with a Flux-Weakening Control Strategy", Journal of Applied Physics, vol. 07, no. 109, (2011).

[34] X. Zhang and C. W. Zhang, "PWM Rectifier and its Control", Beijing: Mechanical Industry Press, (2013).

[35] G. G. Yan, L. Qi and J. H. Li, "PI Controller Parameters Setting of the Three-Phase Decoupling Feedback Linearization VSR System”, Southern Power System Technology, vol. 3, no. 103, (2009).

[36] G. H. Zhou and J. P. Xu, "A Review of Modulation and Control Techniques for Switching Converters", Proceedings of the CSEE, vol. 34, no. 815, (2014).

[37] Z. H. Xu, "Technology Solutions of Control Electric Apparatus Service in Energy Management System", Low-voltage apparatus, vol. 1, no. 14, (2013).

[38] G. G. Yan, L. Qi and J. H. Li, "Implementation of VSC based controllable interruption generator", Advanced Technology of Electrical Engineering and Energy, vol. 29, no. 67, (2010).

[39] L. Qi, "The Research and Development of Power Interruption Generator Based on VSC", A Dissertation for the Degree of Master Eng. Jilin: Northeast Dianli University, (2010).

[40] Paulo Mario dos Santos Dias de Moraes and J. P. Arnaldo, "An Electronic Control Unit for Reducing Contact Bounce in Electromagnetic Contactors", IEEE Transaction on industrial electronics, vol. 55, no. 861, (2008).

[41] W. G. Morsi and M. E. El-Hawary, "A New Perspective for the IEEE Standard 1459-2000 Via Stationary Wavelet Transform in the Presence of Nonstationary Power Quality Disturbance", IEEE Transactions on Power Delivery, vol. 23, no. 2356, (2008).

[42]S. H. Lin and Z. H. Xu, "Performance Characteristics of AC Contactor During Voltage Sag", Proceedings of CSEE, vol. 31, no. 131, (2011).

[43]L. F. Tang and Z. H. Xu, "Design of a New Intelligent Anti-voltage Sag Control Module for AC Contactors", Proceedings of the CSEE, vol. 32, no. 95, (2012).

\section{Authors}

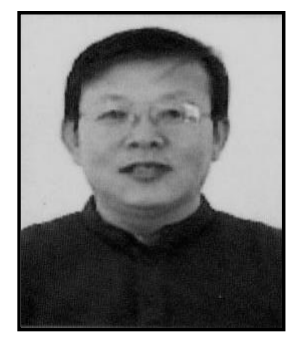

Ming Zong received his PH.D degree from Shenyang University of Technology, China, in 2007, and now is professor in school of Electrical Engineering of Shenyang University of Technology. His research interests include intelligent electrical apparatus, special motor, and advanced control technology.

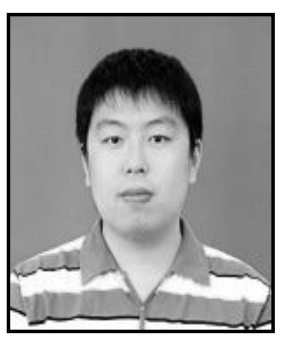

Lei Qi received his M.E degree from Northeast Dianli University in 2010, and now is a Engineer in Liaoning Provincial Institute of safety science china and a PH.D candidates in Shenyang University of Technology. His research interests include intelligent electrical apparatus and its automation. 


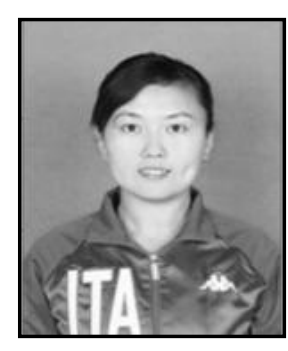

Xuejie Wang received her M.E degree from Northeast Dianli University in 2010, and now is a Engineer in Electrical Engineering department of Shenyang Institute of Engineering and a PH.D candidates in Harbin Institute of Technology. Her research interests include intelligent electrical apparatus and its automation.

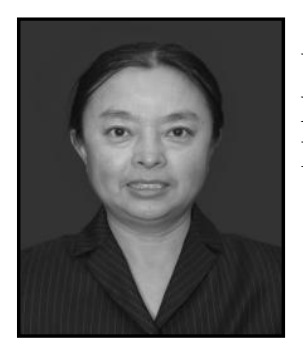

Ying Zhang received her M.E degree from Northeastern University, China, in 1998 and now is a professor in Electrical Engineering department of Shenyang Institute of Engineering. Her research direction is power system automation technology.

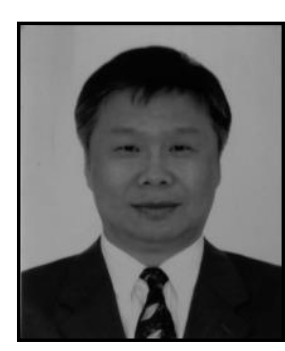

Shenghui Wang received his PH.D degree from Shenyang University of Technology, China, in 1999, and now is professor in Electrical Engineering department of Shenyang Institute of Engineering. His research interests include Application of PV Systems, and also electrical engineering control and automation. 